\title{
Response from the International Transporter Consortium
}

\section{Caroline A. Lee, Kathleen M. Hillgren, Lei Zhang and Joseph W. Polli, on behalf of the International Transporter Consortium}

We are grateful for the comments on our article (Membrane transporters in drug development. Nature Rev. Drug Discov. 9, 215-236 (2010)) $)^{1}$ from Shi, Zhang and Yeleswaram (The relevance of assessment of intestinal P-gp inhibition using digoxin as an in vivo probe substrate. $31 \mathrm{Dec} 2010$ (doi:10.1038/nrd3028-c1) $)^{2}$ regarding their assessment of the utility of digoxin as an in vivo probe for $\mathrm{P}$-glycoprotein ( $\mathrm{P}$-gp). Overall, the International Transporter Consortium (ITC) is in agreement with their comments and supports future research to identify better in vivo probes for P-gp. Below are further thoughts from the ITC on some of the salient points highlighted by Shi and colleagues.

Utility of digoxin as an in vivo probe. The ITC Review did highlight (in the "Decision trees for P-glycoprotein or BCRP inhibitor interactions" (Box 3)) a number of the same limitations of digoxin as a clinical probe as those pointed out by Shi and colleagues. For instance, digoxin was noted as a "unique drug" and may not be the best reference for decisions regarding interaction studies with other P-gp substrate drugs. However, owing to its narrow therapeutic index, interactions that alter the area under the curve (AUC) for digoxin $\geq 1.25$-fold, whether driven by P-gp, other transporters or solubility enhancement, are clinically important for digoxin, as dose adjustment may be warranted. The ITC advised readers to be cautious and not to over-extrapolate the significance of P-gp drug-drug interactions based on the importance of these findings for digoxin.

Further guidance was also given that, if the new molecular entity (NME) is a pure P-gp inhibitor, no drug-drug interaction (DDI) studies may be required other than for clarifying a digoxin dose adjustment requirement, depending on the therapeutic area of the NME and its drug-labelling considerations. It is of crucial importance that the decision to conduct a clinical P-gp DDI study is based on an integration of all the nonclinical and clinical data for the NME. In summary, because digoxin has a narrow therapeutic index and it is a substrate for intestinal P-gp (discussed below), the ITC thinks that clinical DDI studies with drugs that are inhibitors of P-gp should be considered as described in the decision tree.

Importance of solubility and formulation on digoxin absorption. The ITC did mention the importance of considering the digoxin formulation to use in the clinical DDI study, as the different formulations of digoxin have different bioavailability values. As pointed out by the ITC and Shi and colleagues, the standard digoxin tablet has an absolute bioavailability of $\sim 70 \%$, whereas bioavailability for the liquid-filled formulation (that is, the Lanoxicaps capsule) is $\sim 90 \%$. It is of note that the Lanoxicaps capsule product has not been commercially available in the US market since March 2008 (REF. 3); the formal withdrawal due to commercial reasons was requested in July 2010.

Shi and colleagues present an interesting position that a clinical interaction study with digoxin should be mandatory for any investigational drug product with an oral formulation containing solubility-enhancing excipients, regardless of whether the investigational drug product is a P-gp inhibitor, because they hypothesize that interactions observed with digoxin may have little to do with P-gp inhibition but more to do with solubilization of digoxin by the coformulated agents (for example, polyethylene glycol (PEG) or propylene glycol (PG)). However, Shi and colleagues did not provide detailed data in their letter to support their position. The effect of formulations on the pharmacokinetics of digoxin or other narrow therapeutic drugs was beyond the scope of the ITC report, and so the ITC does not have a formal position on this topic.

However, the US Food and Drug Administration, independent of the ITC, has been investigating the potential formulation effect by collecting information on the digoxin formulation (Lanoxin tablet versus Lanoxicaps capsule) and interacting drug formulation (PEG- or PG-containing versus non-PEG- or non-PG-containing). Their preliminary results (L. Zhang, personal communication) suggest a lack of correlation between the presence of PEG or PG in the inhibitor drug formulation and positive inhibition effect on digoxin. For example, some known non-inhibitors of digoxin, such as clopidogrel and rosiglitazone, contain PEG in their formulations, suggesting that PG or PEG in the drug formulation might not be a key factor influencing a digoxin drug interaction. The findings of this work will be reported in a future publication.

Digoxin and intestinal P-gp. Digoxin is a poor/moderately permeable $\mathrm{P}$-gp substrate $^{4}$. Shi et al. noted that "there is no compelling example that we could find in the literature to clearly substantiate clinical drug interaction for digoxin due to inhibition of intestinal P-gp". The ITC would like to highlight the study by Drescher et al. ${ }^{5}$ in which digoxin dosed intravenously in humans was eliminated into the jejunum (measured via an intestinal catheter) and bile. In this study, digoxin intestinal elimination was reduced by approximately $50 \%$ by quinidine (a P-gp inhibitor) and increased by $100 \%$ after multiple-dose rifampicin (a P-gp inducer) treatment, demonstrating a role for intestinal $\mathrm{P}-\mathrm{gp}^{5-7}$. Another indicator for intestinal P-gp interaction is based on pharmacokinetic changes in which maximum plasma concentration $\left(C_{\max }\right)$ and area under the curve (AUC) of oral digoxin increased more than intravenous digoxin, and renal clearance for oral digoxin did not change with the coadministration of amiodarone, verapamil, carvedilol or atorvastatin ${ }^{8-12}$.

Shi et al. further noted that there are other P-gp inhibitor examples such as grapefruit juice and ketoconazole that do not alter the exposure of digoxin. However, although grapefruit juice did not change digoxin exposure, there was a change in the rate of absorption $\left(k_{\mathrm{a}} \text { and } t_{\mathrm{lag}}\right)^{13}$. Also the effect of grapefruit juice on intestinal P-gp will be variable among brands and is known to be dependent on the concentration and the preparation ${ }^{14-16}$. In the case of ketoconazole, it should be given with Coca-Cola, an acidic beverage, for complete dissolution ${ }^{17,18}$. Therefore, the lack of effect of ketoconazole may be attributed to poor dissolution leading to suboptimal concentrations to inhibit intestinal P-gp.

Utility of talinolol as an in vivo probe. Shi and colleagues, along with others, have proposed talinolol as a potential P-gp clinical probe substrate based on this drug being a good in vitro P-gp substrate, having an 
oral bioavailability of $\sim 55 \%$, and negligible metabolic clearance. It also has been shown to exhibit a clinical DDI with erythromycin and rifampin ${ }^{19,20}$. Furthermore, the safety margin is wider for talinolol than for digoxin. These properties make talinolol a potential P-gp probe drug candidate.

However, talinolol has a number of limitations; many of these are similar to digoxin, while others unique to talinolol itself. For example, talinolol is a substrate of multiple transporters, and its pharmacokinetics from the fast-disintegrating dosage form is associated with a double absorption peak that could confound interpretation of a DDI. The pharmacokinetic interactions due to P-gp inhibition/induction seem to be fairly modest, with changes of only $\sim 30-50 \%$ (notably lower than those with digoxin), questioning whether this change is sensitive enough to assess drug interactions; it is noteworthy that cytochrome $\mathrm{P} 450$ probe substrates tend to have much large changes (> 200\%) when co-dosed with an inhibitor. There are also a number of other modest interactions ( 20-35\%) with unknown mechanisms; for example, the P-gp inhibitor verapamil reduces exposure whereas one would have predicted an increase in exposure ${ }^{21}$. Most importantly, talinolol is only approved in Germany, which limits the utility of the drug in other countries including the United States. These limitations do not preclude the use of talinolol as a P-gp probe, but are key considerations when designing a clinical P-gp DDI study, in particular if one considers how the information obtained with talinolol can be extrapolated to other P-gp substrates.
In conclusion, the ITC thanks Shi and colleagues for their thoughtful comments and we agree that future research is warranted to identify better probe substrates for P-gp. We encourage all academic centres and pharmaceutical companies to share data openly to advance the science, knowledge and tools in the area of drug transport.

Caroline A. Lee is at Pfizer Global Research and Development, La Jolla Laboratories, 10724 Science Center Drive, San Diego, California 92121, USA. e-mail: caroline.lee01@pfizer.com

Kathleen M. Hillgren is at Lilly Research Laboratories, Lilly Corporate Center, Indianapolis, Indiana 46285, USA.e-mail: hillgren kathleen m@lilly.com

Lei Zhang is at the Office of Clinical Pharmacology, Office of Translational Sciences, Center for Drug Evaluation and Research, Food and Drug Administration, 10903 New Hampshire Avenue, Silver Spring, Maryland 20993-0002, USA. e-mail: leik.zhang@fda.hhs.gov

Joseph W. Polli is at PO Box 13398, GlaxoSmithKline, Research Triangle Park, North Carolina 27709, USA. Correspondence to J.W.P. e-mail: joseph.w.polli@gsk.com doi: $10.1038 / \mathrm{nrd3028-c2}$

1. The International Transporter Consortium. Membrane transporters in drug development. Nature Rev. Drug Discov. 9, 215- 236 (2010).

2. Shi, J. G., Zhang, Y. \& Yeleswaram, S. The relevance of assessment of intestinal P-gp inhibition using digoxin as an in vivo probe substrate. Nature Rev. Drug Discov. 31 Dec 2010 (doi: 10.1038/nrd3028-c1).

3. American Society of Health-System Pharmacists. Digoxin oral products [online], < http://www.ashp.org Import/PRACTICEANDPOLICY/PracticeResource Centers/DrugShortages/GettingStarted/ ResolvedShortages/bulletin.aspx?id = $405>$ (2009)

4. Keogh, J. P. \& Kunta, J. R. Development, validation and utility of an in vitro technique for assessment of potential clinical drug-drug interactions involving P-glycoprotein. Eur. J. Pharm. Sci. 27, 543-554 (2006)

5. Drescher, S. et al. P-glycoprotein-mediated intestinal and biliary digoxin transport in humans. Clin. Pharmacol. Ther. 73, 223-231 (2003).
6. Greiner, B. et al. The role of intestinal P-glycoprotein in the interaction of digoxin and rifampin. J. Clin. Invest. 104, 147-153 (1999).

7. Eichelbaum, M. et al. The role of intestinal P-glycoprotein in the interaction of digoxin and rifampin. J. Clin. Invest. 110, 571 (2002).

8. Fenner, K. S. et al. Drug-drug interactions mediated through P-glycoprotein: clinical relevance and in vitroin vivo correlation using digoxin as a probe drug. Clin. Pharmacol. Ther. 85, 173-181 (2009).

9. Boyd, R. A. et al. Atorvastatin coadministration may increase digoxin concentrations by inhibition of intestinal P-glycoprotein-mediated secretion. J. Clin. Pharmacol. 40, 91-98 (2000).

10. De Mey, C., Brendel, E. \& Enterling, D. Carvedilol increases the systemic bioavailability of oral digoxin Br. J. Clin. Pharmacol. 29, 486-490 (1990).

11. Robinson, K. et al. The digoxin-amiodarone interaction. Cardiovasc. Drugs Ther. 3, 25-28 (1989)

12. Rodin, S. M., Johnson, B. F., Wilson, J., Ritchie, P. \& Johnson, J. Comparative effects of verapamil and isradipine on steady-state digoxin kinetics. Clin. Pharmacol. Ther. 43, 668-672 (1988).

13. Parker, R. B., Yates, C. R., Soberman, J. E. \& Laizure, S C. Effects of grapefruit juice on intestinal P-glycoprotein: evaluation using digoxin in humans. Pharmacotherapy 23, 979-987 (2003).

14. Ohnishi, A. et al. Effect of furanocoumarin derivatives in grapefruit juice on the uptake of vinblastine by $\mathrm{Caco}$ 2 cells and on the activity of cytochrome P450 3A4. Br. J. Pharmacol 130, 1369-1377, (2000).

15. de Castro, W. V., Mertens-Talcott, S., Derendorf, H. \& Butterweck, V. Grapefruit juice-drug interactions: Grapefruit juice and its components inhibit P-glycoprotein (ABCB1) mediated transport of talinolo in Caco-2 cells. J. Pharm. Sci. 96, 2808-2817 (2007).

16. Dahan, A. \& Amidon, G. L. Grapefruit juice and its constituents augment colchicine intestinal absorption potential hazardous interaction and the role of p-glycoprotein. Pharm. Res. 26, 883-892 (2009).

17. Chin, T. W., Loeb, M. \& Fong, I. W. Effects of an acidic beverage (Coca-Cola) on absorption of ketoconazole. Antimicrob. Agents Chemother. 39, 1671-1675 (1995).

18. Hurwitz, A., Ruhl, C. E., Kimler, B. F., Topp, E. M. \& Mayo, M. S. Gastric function in the elderly: effects on absorption of ketoconazole. J. Clin. Pharmacol. 43, 996-1002 (2003).

19. Schwarz, U. I., Gramatte, T., Krappweis, J., Oertel, R. \& Kirch, W. P-glycoprotein inhibitor erythromycin increases oral bioavailability of talinolol in humans. Int J. Clin. Pharmacol. Ther. 38, 161-167 (2000).

20. Westphal, K. et al. Induction of P-glycoprotein by rifampin increases intestinal secretion of talinolol in human beings: a new type of drug/drug interaction. Clin. Pharmacol. Ther. 68, 345-355 (2000).

21. Toffoli, G. et al. Structure-activity relationship of verapamil analogs and reversal of multidrug resistance. Biochem. Pharmacol. 50, 1245-1255 (1995). 